\section{Mascarillas: ¿falsa seguridad o falso temor?}

\section{Masks: false security or false fear?}

\section{Señor Editor:}

Durante la pandemia de gripe de 1919 el profesor George H. Weaver advirtió que "el uso de las mascarillas no debía dar una sensación de seguridad infundada a aquellos que los utilicen, llevándolos a abandonar las otras medidas de prevención para reducir el contagio". Un siglo después la Organización Mundial de la Salud también alertó dentro de los riesgos, de utilizar mascarillas al advertir sobre "la falsa sensación de seguridad que pueden conferir, lo cual podría llevar al incumplimiento de otras medidas profilácticas, como el distanciamiento físico y la higiene de las manos"2. Sin embargo, la ausencia de referencias bibliográficas para respaldar esta afirmación en sus recomendaciones sobre el uso de mascarillas en el contexto de la actual pandemia (COVID-19) ocasionada por el SARS-CoV-2, hace cuestionarnos si esto se debe a que nadie ha podido proveer una evidencia concreta respecto a este potencial riesgo. Además, hay una sorprendente contradicción entre las recomendaciones provistas para la presente pandemia con las sugeridas por el Programa Mundial de la Gripe, en las cuales no se realiza esta advertencia en relación a los riesgos en el uso de mascarillas ${ }^{3}$. Es particularmente intrigante que en dicho documento se plantea una posible correlación positiva entre el uso de mascarillas con otras medidas de prevención ${ }^{4}$.

No es claro por qué el efecto de "falsa seguridad" ha sido incorporado en las recientes recomendaciones, pero investigaciones recientes muestran que no estaría basado en evidencia científica ${ }^{5,6} \mathrm{y}$, por lo tanto, debería ser reconsiderado.

La concepción habitual de la "compensación de riesgo" se fundamenta en la creencia que las personas tienen un nivel de riesgo con el que se sienten conformes y ajustan su conducta para mantenerlo, pero la evidencia es más consistente con que las personas que adoptan una conducta de cuidado son en realidad más permeables, y no menos, a incorporar otras medidas similares que incrementen su seguridad.

En última instancia, el verdadero peligro de acuerdo a investigaciones previas, es que la inconsistencia o ambigüedad del discurso puede llevar a las personas a cuestionar la credibilidad de la información generada por prestigiosas instituciones y en última medida recibida por la población en general ${ }^{7}$.

Finalmente, esto repercute en la escasez de recursos tanto farmacológicos como no farmacológicos para lidiar con la infección por SARS-CoV-2, lo cual hace que una comunicación efectiva se transforme en un pilar fundamental del abordaje de la pandemia.

Pablo Richly ${ }^{1}$ y Walter D. Cardona Maya ${ }^{2}$. ${ }^{I}$ CESAL - Centro de Salud Cerebral. Buenos Aires, Argentina.

${ }^{2}$ Grupo Reproducción, Facultad de Medicina, Universidad de Antioquia, Medellín, Colombia.

\section{Referencias bibliográficas}

1.- Weaver G. Droplet infection and its prevention by the face mask. J Infect Dis 1919; 24:218-30. doi: 10.1093/ infdis/24.3.218

2.- World Health Organization. Advice on the use of masks in the context of COVID-19: interim guidance, 5 june 2020. (Fecha de acceso: 2 de agosto de 2020).

3.- World Health Organization. Non-pharmaceutical public health measures for mitigating the risk and impact of epidemic and pandemic influenza; 2019. (Fecha de acceso: 2 de agosto de 2020).

4.- Wada K, Oka-Ezoe K, Smith D R. Wearing face masks in public during the influenza season may reflect other positive hygiene practices in Japan. BMC Public Health 2012; 12: 1065. doi: 10.1186/1471-2458-12-1065.

5.- Bakhit M, Krzyzaniak N, Scott A M, Clark J, Glasziou P, Del Mar Ch. Downsides of face masks and possible mitigation strategies: a systematic review and meta-analysis. medRxiv 2020.06.16.20133207. doi: 10.1101/2020.06.16.20133207.

6.- Mantzari E, Rubin G J, Marteau T M. Is risk compensation threatening public health in the covid-19 pandemic? BMJ 2020; 370: m2913. doi: 10.1136/bmj.m2913.

7.- The Royal Society. Face masks and coverings for the general public: Behavioural knowledge, effectiveness of cloth coverings and public messaging. 26 June 2020. Disponible en: https://royalsociety.org/-/media/policy/projects/set-c/ set-c-facemasks.pdf?la=en-GB\&hash=A22A87CB28F7D6A D9BD93BBCBFC2BB24. (Fecha de acceso: 2 de agosto de 2020). 